\title{
A NOTE ON THE PSEUDO-ARC
}

BY

\author{
EDWIN E. MOISE
}

The author has given an example $\left({ }^{1}\right)$ of a compact indecomposable continuum (the pseudo-arc) which is homeomorphic to each of its non-degenerate subcontinua. R. H. Bing has shown $\left({ }^{2}\right)$ that this continuum is homogeneous. Following is a proof of Bing's result, based on an extension of the author's previous paper, using chains with overlapping links, as mentioned conversationally by Bing.

A chain $D$ is a quasi-refinement of a chain $C$ if each link of $D$ lies in the interior of the closure of the sum of two adjacent links of $C$.

Lemma. Let $C$ and $D$ be chains from $P$ to $Q$, and let $D$ be very crooked $\left({ }^{3}\right)$ with respect to $C$. Let $d$ be a link of $D$. Then there is a quasi-refinement $E$ of $C$ which is a consolidation of $D$, such that one end-link of $E$ contains $d$ and the other is an end-link of $D$.

Proof. We may assume in the proof that $D$ is its own amalgam with respect to $C$; the theorem will then follow a fortiori. Let $k$ be the number of links of $C$, and suppose that the theorem is true for all shorter chains $C$. If $k$ is less than five, the lemma is obvious; otherwise, $D$ is the sum of three chains $D_{1}$ from $d_{1}$ to $d_{h}, D_{2}$ from $d_{h}$ to $d_{i}$, and $D_{3}$ from $d_{i}$ to $d_{j}$, such that $D_{1}-d_{1}$ and $D_{3}-d_{j}$ are each similar to $D_{2}$ with respect to $C$, under transformations $T_{1}$ and $T_{3}$, the other transformations in each case being the identity for each link of $C$. Now (1) suppose that $d$ belongs to $D_{1}$. By hypothesis, there is a chain $E_{1}$ which satisfies the conclusion of the theorem with respect to $D_{1}$ and $C-c$, where $c$ is the last link of $C$. Now if $d_{1}$ is an end-link of $E_{1}$, add $d_{2}$ to this end-link; and for each link $x$ of $D_{1}-d_{1}$, add $T_{1}^{-1}(x)$ to the link of $E_{1}$ that contains $x$. Let the resulting chain be $E_{1}^{\prime}$. Let $E$ be the chain whose links are (i) the links of $E_{1}^{\prime}$, and (ii) the links of $D_{3}-d_{i}$. On the other hand, if $d_{h}$ is an end-link of $E_{1}$, let $E$ be the chain whose links are (i) the links of $E_{1}$, and (ii) the links of $D_{2}+D_{3}+d_{i}$. A precisely analogous procedure is used if $d$ belongs to $D_{3}$.

(2) If $d$ belongs to $D_{2}$, suppose that $E_{1}$ has $d_{i}$ in its end-link which does not contain $d$. Add $d_{1}+d_{2}$ to the end-link of $E_{1}$ which contains $d_{i}$, and for each $x$ of $D_{2}$, add $T_{2}(x)$ to the link of $E_{1}$ that contains $x$. This gives a chain $E_{1}^{\prime}$.

Presented to the Society, February 26, 1949; received by the editors June 21, 1948.

(1) An indecomposable plane continuum which is homeomorphic to each of its non-degenerate subcontinua, Trans. Amer. Math. Soc. vol. 63 (1948) pp. 581-594.

(2) R. H. Bing, A homogeneous indecomposable plane continuum, Duke Math. J. vol. 15 (1948) pp. 729-742.

(3) For definitions of this term and others used below, see footnote 1. 
Let $E$ be the chain whose links are the links of $E_{1}^{\prime}$ and the links of $D_{3}-d_{i}$.

From the lemma it follows that for each point $R$ of $M$ there is a sequence $D_{j_{1}}, D_{j_{2}}, \cdots$ of chains such that (1) each $D_{j_{i}}$ is a chain from $R$ to $P$ or $Q$, (2) $D_{j_{i+1}}$ is a consolidation of $C_{j_{i+1}}$ and a quasi-refinement of $C_{j_{i+1+2}}$ and of $D_{j_{i}}$. Since one of the possibilities in (1) must occur infinitely many times, we may assume that each $D_{j_{i}}$ is a chain from $R$ to $S$, where $S$ is $P$ or $Q$.

Given the sequence $D_{j_{1}}, D_{j_{2}}, \cdots$, we wish to obtain a corresponding sequence $D_{j_{1}}^{\prime}, D_{j_{2}}^{\prime}, \ldots$ in which each term after the first is a refinement of its predecessor. This may be done in the following manner: Given a pair $c, c^{\prime}$ of adjacent links of $D_{j_{1}}$, and a link of $D_{j_{2}}$ which intersects both $c$ and $c^{\prime}$, we either (1) add $d$ to $c$ and subtract $\bar{d} \cdot c^{\prime}$ from $c^{\prime}$, or (2) add $d$ to $c^{\prime}$ and subtract $\bar{d} \cdot c$ from $c$. This gives a chain $D_{j_{1}, 1}$, which has $D_{j_{2}}$ as a refinement. Analogous operations on $D_{j_{2}}$ give $D_{j_{2}, 1}$, which has $D_{j_{3}}$ as a refinement; we obtain $D_{j_{1}, 2}$ by performing a similar operation on $D_{j_{1}}$ and $D_{j_{2}, 1}$, with the proviso that the choice of (1) or (2) is to be made in the same way for a link of $D_{j_{2}, 1}$ as it was made for the corresponding link of $D_{j_{2}}$.

By repeating this process indefinitely, we obtain, for each link $c$ of each $D_{j_{i}}$, a convergent sequence of sets $c_{1}, c_{2}, \cdots$. We let the chains $D_{j 1}^{\prime}, D_{j 2}^{\prime}, \cdots$ be the chains whose links are the interiors of the closures of the sets $\lim _{n \rightarrow \infty} c_{n}$.

The $D^{\prime}$-sequence has all the properties required by Definition 7 of the paper cited in footnote 1 , except that in (3) and (5) we must replace the phrase "five links" by the phrase "at least five links," and that we must replace the first part of condition (2) by the condition that each $D_{j_{i+1}}^{\prime}$ has a proper consolidation with respect to $D_{j_{i}}^{\prime}$ which is very crooked with respect to $D_{j_{i}}^{\prime}\left({ }^{4}\right)$. That is to say, loosely speaking, the $D^{\prime}$-sequence differs from the $Y$-sequence only in that its chains are more finely subdivided and oscillate more. We observe that the $D^{\prime}$-sequence is its own $C$-sequence in the sense of Definition 10. Theorems 9, 11, and 12 of the paper cited in footnote 1 therefore apply, the required changes in the proofs being entirely trivial. It follows that for each $R$ of $M$ there is a homeomorphism of $M$ onto itself throwing $R$ into $P$ or $Q$ and leaving either $P$ or $Q$ fixed. There is obviously a homeomorphism of order 2 which interchanges $P$ and $Q$. Therefore, $M$ is homogeneous.

UNIVERSITY OF MiCHIGAN

ANN ARbor, Mich.

(4) Or we may use the more evident fact that each term $D_{n}^{\prime}$ of the $D^{\prime}$-sequence is exceedingly crooked with respect to its predecessor $D_{m}^{\prime}$, in the sense that if the subchain $D_{n}^{\prime \prime}$ of $D_{n}^{\prime}$ is a refinement of the subchain $D_{m}^{\prime \prime}$ of $D_{m}^{\prime}$, and has an end-link in each end-link $d_{1}, d_{k}$ of $D_{m}^{\prime \prime}$, then $D_{n}^{\prime \prime}$ is the sum of three chains having only their end-links in common, and having their end-links in $d_{1}$ and $d_{k-1}, d_{k-1}$ and $d_{2}$, and $d_{2}$ and $d_{k}$ respectively. This may be proved in a straightforward manner, using condition (2) for the $D$-sequence. 